\title{
Correlation of Isokinetic Testing and ACL Failure With the Short Graft Tape Suspension Technique at Six Months
}

\author{
Mathieu Severyns, M.D., M.Sc., Stéphane Plawecki, M.D., Ph.D., \\ Guillaume-Anthony Odri, M.D., Ph.D., Tanguy Vendeuvre, M.D., Ph.D., \\ Frédéric Depiesse, M.D., Jean-Francois Flez, M.D., and Louis-Antoine Liguori, M.D.
}

\begin{abstract}
Purpose: The objective of this study was to correlate the data of the 6-month postoperative isokinetic muscle evaluation before resuming sports activities with the occurrence of ACL reconstruction rerupture after semitendinosus short graft. Methods: From 2015 to 2018, all patients who were operated for an ACL reconstruction with a short semitendinosus autograft (TLS System) and who performed isokinetic tests on dynamometer at their 6th postoperative month were included in this study. The follow-up was prospective with the measurement of epidemiological, radiographic, and isokinetic parameters at 6 months of the ACL reconstruction. The cohort was divided into 2 groups: one group without an ACL reconstruction rerupture (Group 1) and the second group with a rerupture (Group 2). Results: One hundred and four patients were analyzed with an average follow-up of 42.3 months (Minimum: 24; Maximum: 63.5), of which 11 patients $(10.6 \%)$ had an ACL reconstruction rerupture. Group 1 consisted of 93 patients with an average age of $26.5 \pm 9.0$ years old who did not have an ACL reconstruction rerupture with an average follow-up of $41.6 \pm 12.1$ months. Group 2 consisted of 11 patients with an average age of $22.7 \pm 6.1$ years old, who had an ACL reconstruction rerupture with an average follow-up of $44.8 \pm 11.3$ months. Concerning extension force recovery, the ratio between operated and healthy knee was $81.8 \% \pm 32.0$ for Group 1 , and $53.4 \% \pm 20.6$ for Group $2(P=.035)$. A statistically significant difference was also found $(P=.0017)$ during $60^{\circ} / \mathrm{s}$ flexion isokinetic test between the two groups. Conclusions: This study revealed a significant link between muscle weakness in flexion and extension during $60^{\circ} / \mathrm{s}$ isokinetic test at 6 months of ACL reconstruction and semitendinosus autograft rerupture. Patients with an ACL reconstruction retear had inferior muscle dynamometric recovery results at 6 months before resuming sports activities. Level of Evidence: Level III, prognostic, retrospective cohort study.
\end{abstract}

\section{Introduction}

$\mathbf{T}$ he rates of rerupture after anterior cruciate ligament reconstruction are of the order of 1.7 to $10.3 \%$ when considering studies with a minimum 3-year follow-up in cases of semitendinosus/gracilis

From the Department of Orthopaedic surgery, University Hospital of Martinique, Fort-de-France, France (M.S., S.P., G.A.O.); Prime Institut, Centre National Recherche Scientifique, University of Poitiers, Poitiers, France (M.S., T.V.); Department of Clinical and Exercise Physiology, University Hospital of Martinique, Fort-de-France, France (F.D.); and Department of Orthopaedic Surgery, Clinique Saint Paul, Fort-de-France, France (J.-F.F., L.-A.L.).

Full ICMJE author disclosure forms are available for this article online, as supplementary material.

Received August 8, 2021; accepted November 13, 2021

Address correspondence to Mathieu Severyns, M.D., M.Sc., Hôpital Pierre Zobda Quitman, 97261 Fort-de-France Cedex, France. E-mail: mathieu. severyns@hotmail.com

(C) 2021 THE AUTHORS. Published by Elsevier Inc. on behalf of the Arthroscopy Association of North America. This is an open access article under the CC BY-NC-ND license (http://creativecommons.org/licenses/by-nc-nd/4.0/). 2666-061X/211115

https://doi.org/10.1016/j.asmr.2021.11.020 tendons autograft $(\mathrm{ST} / \mathrm{G}) \cdot{ }^{1-8}$ Although mispositioning of bone tunnels is the primary cause of anterior cruciate ligament (ACL) reconstruction rerupture by positive or negative anisometry ranging from $52.2 \%$ to $95.6 \%,{ }^{9-13}$ the number of risk factors for rerupture are clearly identified in the literature, including genu varum, posterior tibial slope, anterior tibial subluxation and intercondylar notch size. ${ }^{14-18}$ While data on muscle recovery and return-to-sport (RTS) are very well documented. ${ }^{19-22}$ very few studies have done a quantitative analysis of the link between muscle deficit and ACL reconstruction retear. The objective of this study was to correlate the data of the 6-month postoperative isokinetic muscle evaluation before resuming sports activities with the occurrence of ACL reconstruction rerupture after semitendinosus short graft. The hypothesis was that patients with an ACL reconstruction retear had inferior muscle dynamometric recovery results at 6 months compared to those without this complication. 


\section{Method}

\section{Study Population}

This is a retrospective cohort study. From June 2015 to December 2018, one surgeon performed ACL reconstruction with a short semitendinosus autograft for all patients who required ACL reconstruction (using TLS ${ }^{\circledR}$ (Tape locking screw) system (FH Orthopaedics, Heimsbrunn, France). All patients who had ACL reconstruction and performed isokinetic tests at their 6th postoperative month were included in this study.

The inclusion criteria were adult patient $(>18$ years old), who initially had an ACL tear without rupture of a peripheral plane and benefited from a primary ACL reconstruction surgery without anterolateral ligament reconstruction (ALLR) or lateral extra-articular tenodesis (LET) and who performed an isokinetic muscle test on dynamometer at the 6th postoperative month as part of the care protocol. The exclusion criteria were reconstruction for ACL reconstruction rerupture, multiligament surgery, no isokinetic assessment on dynamometer, and refusal to participate in this study. The rehabilitation protocol was standardized and given to the patient and the physiologist. Full weight bearing was immediately authorized, and the recovery of passive and active motions and passive strengthening was started the next day with the physiologist. The RTS was allowed after 6 months if the patient passed the isokinetic test with an operated/healthy knee ratio higher than $85 \%$ in flexion and extension.

\section{Surgical Technique}

The Tape Locking Screw (TLS system; FH Orthopaedics, Heimsbrunn, France) system is an ACL reconstruction method created in 2003. ${ }^{23}$ The surgical technique consists of harvesting only one hamstring tendon (semitendinosus tendon), prepared into a short, four- to five-strand closed loop, and with a 500-N preload. According to the surgical technique, the length of the graft is correlated with the patient size. Tibial and femoral tunnels are shorter and created in a retrograde manner. Press-fit of the graft into the bone tunnels is obtained by tension on polyethylene terephthalate strips that are attached to the bone with dedicated $20-\mathrm{mm}$ interference screws. Each patient was operated on with the use of a tourniquet under exclusive general anesthesia as an outpatient.

\section{Isokinetic Test Procedure}

Muscle recovery, analyzed between the healthy and operated knee, was evaluated with isokinetic tests using the CONTREX human dynamometer ${ }^{24}$ by a senior reeducating physician (Fig 1). The evaluation was done systematically in extension (quadriceps muscle), as well as in flexion (hamstrings), starting with the healthy side without prior warmup. Two different speeds were applied, one at $60^{\circ} / \mathrm{s}$ to obtain the muscle power curves and the other at a higher speed $\left(180^{\circ} / \mathrm{s}\right)$ for the muscle response (or the capacity to quickly recruit muscle fibers).

\section{Evaluation Criteria}

The follow-up was prospective with the measurement of epidemiological (age, sex, weight, body mass index, and Tegner score ${ }^{25}$ ), radiographic, and isokinetic parameters.

All of the results of the isokinetic tests on dynamometer performed at 6 months postoperatively of the ACL reconstruction were collected. At a mean of 42.3 months (minimum: 24; maximum: 63.5) of follow-up, the data were collected retrospectively. The cohort was then divided into 2 groups (Fig 2): one group without an ACL reconstruction re-rupture (Group 1) and the second group with a rerupture (Group 2). Graft failure diagnosis was initially clinical, and ACL retear was confirmed by magnetic resonance imaging (MRI). Measurement of the position of the tibial and femoral tunnels (anterior border of the exit holes) were realized in accordance with the ratio method described by Aglietti et al. by two senior surgeons. ${ }^{26,27}$

\section{Statistical Analysis}

Data were collected in an Excel spreadsheet (Microsoft, Richmond, WA) and were analyzed with JMP 10.0 software (SAS, Inc.). A Shapiro-Wilk test was performed to assess the normal distribution of quantitative variables. Comparison of qualitative variables was done with a $\chi^{2}$-test. A Student's $t$-test was performed to compare quantitative variables. The significance threshold was then $P<.05$ for all tests. Mean comparative statistics between operated knee and the healthy knee were calculated for paired samples.

\section{Results}

One hundred and four (104) patients were analyzed including 11 patients $(10.6 \%)$ who had an ACL reconstruction rerupture, with an average follow-up of $42.3 \pm 12.1$ months (minimum: 24; maximum: 63.5). Patients' characteristics, radiographic parameters, and isokinetic tests are summarized in Table 1 .

Group 1 consisted of 93 patients with an average age of $26.5 \pm 9.0$ years old who did not have an ACL reconstruction rerupture with an average follow-up of $41.6 \pm 12.1$ months. Group 2 consisted of 11 patients with an average age of $22.7 \pm 6.1$ years old, who had an ACL reconstruction rerupture with an average followup of $44.8 \pm 11.3$ months (Table 2). The average time to rerupture after ACL reconstruction was 11.45 months (minimum: 7; maximum: 19). All of these reruptures occurred as a result of sports trauma.

No significant differences were found between the two groups concerning the age at surgery, the 


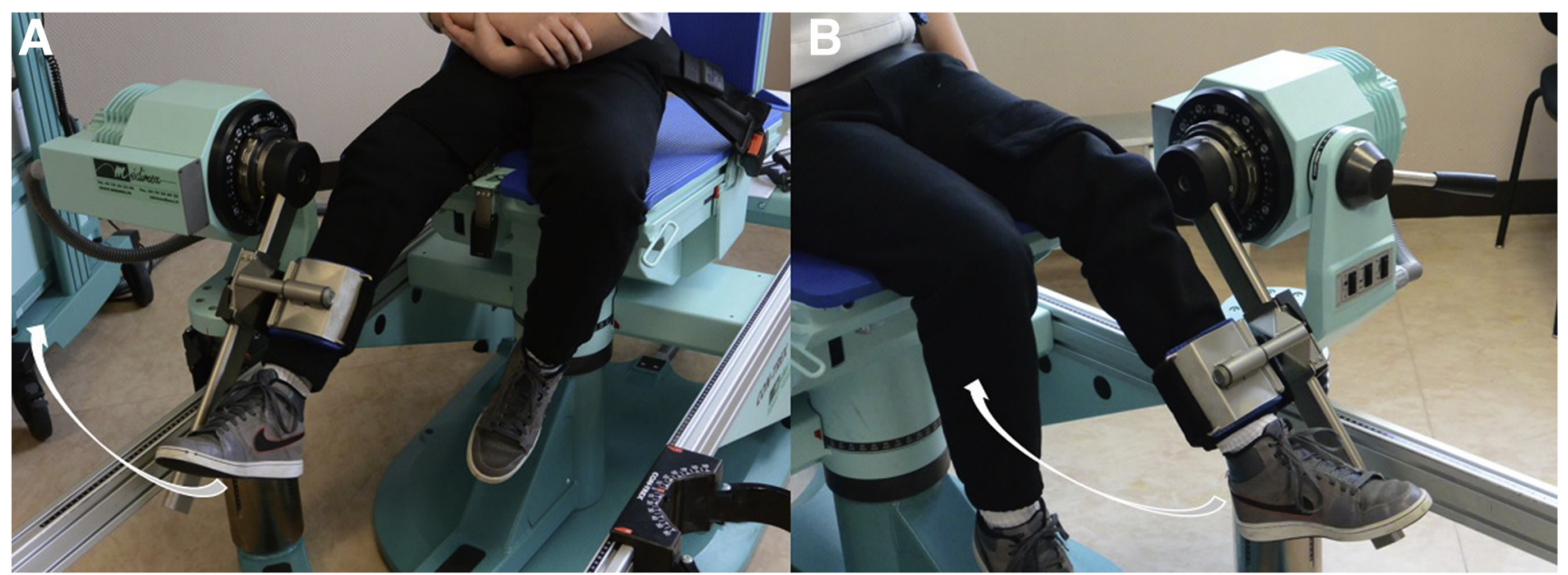

Fig 1. Isokinetic test (CONTREX dynamometer) at 6 months of anterior cruciate ligament reconstruction: example of $60^{\circ} / \mathrm{s}$ strength test on an operated knee (A) and response muscle test at $180^{\circ} / \mathrm{s}$ on a healthy knee (B).

follow-up, gender, weight, and BMI. The preoperative Tegner score was also statistically not significant between the two groups: average of $7 \pm 1.1$ for Group 1 and $7.3 \pm 1.1$ for Group $2(P=.56)$.

Concerning extension force recovery $\left(60^{\circ} / \mathrm{s}\right)$, the ratio between the operated and the healthy knee was $81.8 \% \pm 32.0$ for the Group 1, and $53.4 \% \pm 20.6$ for the Group $2(P=.035)$. A statistically significant difference was also found $(P=.0017)$ during $60^{\circ} / \mathrm{s}$ flexion isokinetic test between the two groups (Table 3 ).

The $180^{\circ} / \mathrm{s}$ muscle response, either on extension or flexion, showed no significant statistical difference. The values recovered were $80.4 \% \pm 22.4$ versus $65.5 \% \pm$ 19.6 for quadriceps muscle $(P=.24)$, and $92.1 \% \pm 20.7$ versus $88.4 \% \pm 15.5$ for hamstrings $(P=.7)$.

For radiograph parameters, no statistical differences were found in terms of tibial $(P=.78)$ and femoral $(P=.89)$ tunnels positioning between the two groups.

\section{Discussion}

The most important finding of this study confirmed that patients with an ACL reconstruction rerupture had lower isokinetic results at 6 months compared to those without this complication in terms of muscular strength $\left(60^{\circ} / \mathrm{s}\right)$, but not concerning the muscle response $\left(180^{\circ} / \mathrm{s}\right)$. Isokinetic tests are commonly used during ACL reconstruction rehabilitation..$^{28-30}$ There is only one study in the literature reporting a decrease in hamstring and quadriceps muscle strength on dynamometer $\left(60^{\circ} / \mathrm{s}\right)$ in professional athletes with an ACL reconstruction re-tear. ${ }^{31}$ Although no threshold study was carried out, this series allowed a quantitative comparison of the muscular deficit on isokinetic tests prospectively before RTS.

Significant differences were found at $60^{\circ} / \mathrm{s}$ during extension and flexion isokinetic tests. This is in accordance with results of other studies that show an

Fig 2. Study enrollment flowchart.

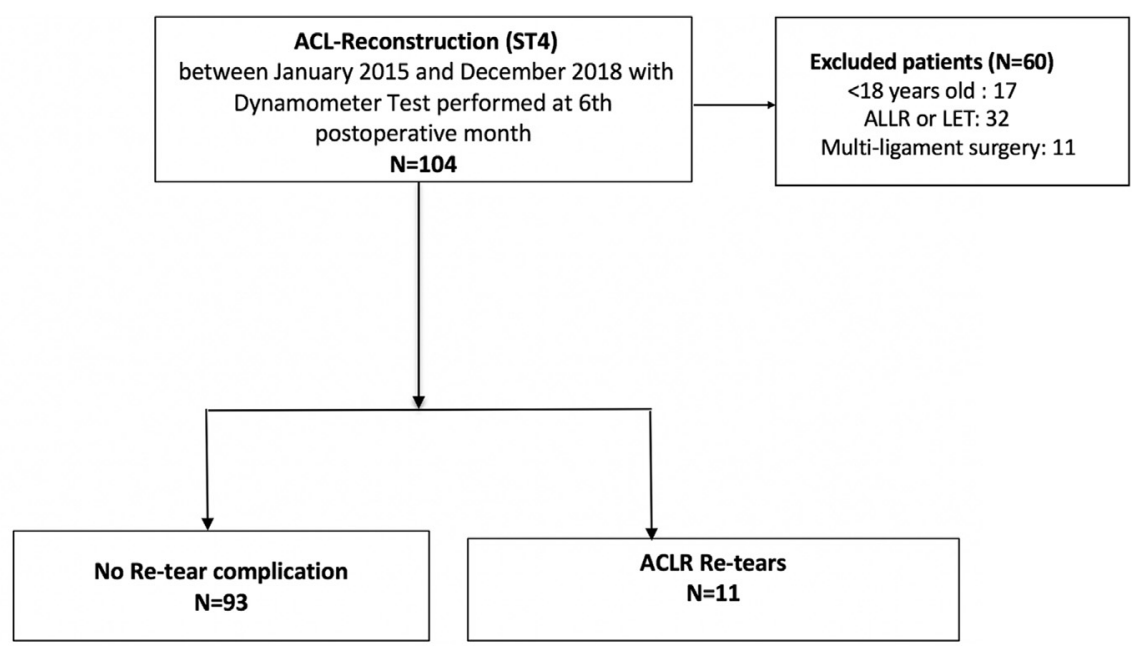


Table 1. Epidemiologic and Radiographic Parameters of Study Population

\begin{tabular}{lc}
\hline \multicolumn{1}{c}{ Parameter } & Value \\
\hline Number of patients & $n=104$ \\
Side (Right/Left) & $46 / 56$ \\
Sex (Female/Male) & $35 / 68$ \\
Weight (kg, median $\pm \mathrm{SD})$ & $73.1 \pm 15.8$ \\
Size (m, median $\pm \mathrm{SD})$ & $1.74 \pm .09$ \\
BMI (median $\pm \mathrm{SD})$ & $23.5 \pm 4.1$ \\
Follow-up (Months, median $\pm \mathrm{SD})$ & $42.3 \pm 12.1$ \\
Preoperative Tegner Score (median $\pm \mathrm{SD})$ & $7.0 \pm 1.1$ \\
Posterior tibial Slope (degrees, median $\pm \mathrm{SD})$ & $10.1 \pm 3.8$ \\
HKA alignment (degrees, median $\pm \mathrm{SD})$ & $177.8 \pm 3.3$ \\
\hline
\end{tabular}

HKA, hip-knee-ankle.

increased operated/healthy knee ratio only at this angular velocity after primary ACL reconstruction. ${ }^{32-34}$ Abundant data exist for these selected velocity and have been shown to highlight strength deficits. Torque output decreases as angular velocity increases above $60^{\circ}$ \% $\mathrm{s}$, and the maximum torque output is shown to be between 0 and $60^{\circ} / \mathrm{s}^{35}$ For this reason, if the angular velocity that is used for strength evaluation is greater than $60 \%$ s, strength deficiencies might not be highlighted.

Conserving the gracilis muscle would have a faster recovery time in flexion when compared to reconstruction requiring the removal of the two hamstrings. ${ }^{19-22}$ Although isolated sampling of semitendinosus was performed, ${ }^{36,37}$ isokinetic results on flexion at 6 months $\left(60^{\circ} / \mathrm{s}\right)$ are still insufficient.

In their systematic review of the literature, BarberWestin et al. pointed out that RTS was based on nonspecific subjective criteria, and in the majority of cases, surgeons set the time criterion for RTS at 6 months, the theoretical date of graft healing. ${ }^{29}$ Although isokinetic tests provide objective data on muscle strength recovery, ${ }^{38}$ literature data do not support these tests as a reliable and reproducible predictor for RTS. ${ }^{28,39}$ Van Grinsven et al. suggest, however, that a threshold of $85 \%$ for the extension and the flexion must be reached before RTS. ${ }^{40}$ In this series, the ratio between operated and healthy knee at $60 \%$ s was $71.6 \%$ for flexion and $53.4 \%$ for extension in Group 2. Therefore, a large majority of patients received instructions to delay their return-tosport and to continue muscle strengthening following their isokinetic tests. For Bobkin et al., ${ }^{41}$ patients demonstrated increasing subjective and quadriceps function when tested at later time points from surgery with a deficit muscle strength at 9 months that might improve with a 2-month rehabilitation protocol. Concerning RTS, Nagai et al. ${ }^{42}$ supported the continued use of isokinetic testing when examining an individual's readiness to return to sport.

\section{Study Limitations}

The main limitation of this study is its reduced size for Group 2. Indeed, the ACL reconstruction retear is a rare event, and it would have been necessary to include a much larger number of subjects to bring more power to this prospective monitoring. Although the groups were homogeneous in terms of epidemiological data, preoperative sport level, and bone tunnel position, the low number of participants in Group 2 did not allow a multivariate analysis. Finally, no threshold study was carried out. It would be interesting to carry out a largerscale prospective multicenter study to find the threshold from which muscle deficit would be predictive of an ACL reconstruction rerupture in case of semitendinosus reconstruction.

\section{Conclusion}

This study revealed a significant link between muscle weakness in flexion and extension during $60^{\circ} / \mathrm{s}$ isokinetic test at 6 months of ACL reconstruction and ACL reconstruction rerupture after semitendinosus short autograft. Patients with an ACL reconstruction retear had inferior muscle dynamometric recovery results at 6 months before resuming sports activities.

Table 2. Patients' Characteristics and Distribution Tests (Student and $\chi^{2}$ ) Between the Two Groups (With and Without ACL Reconstruction Rerupture)

\begin{tabular}{|c|c|c|c|}
\hline & $\begin{array}{l}\text { Without } \\
\text { ACLR Retears } \\
(n=93)\end{array}$ & $\begin{array}{l}\text { With } \\
\text { ACLR Retears } \\
(n=11)\end{array}$ & $P$ \\
\hline $\begin{array}{l}\text { Age, median } \pm \text { SD } \\
\quad \text { (years) }\end{array}$ & $26.5 \pm 9.0$ & $22.7 \pm 6.1$ & .53 \\
\hline Sex & & & .86 \\
\hline Male & 62 & 7 & \\
\hline Female & 31 & 4 & \\
\hline BMI median \pm SD & $23.6 \pm 3.8$ & $21.3 \pm 3.5$ & .22 \\
\hline $\begin{array}{l}\text { Weight, } \\
\text { median } \pm \text { SD }(\mathrm{kg})\end{array}$ & $73.3 \pm 1.6$ & $65.7 \pm 6.1$ & .24 \\
\hline \multicolumn{4}{|l|}{ Side } \\
\hline Right & 44 & 5 & \\
\hline Left & 49 & 6 & .51 \\
\hline $\begin{array}{l}\text { Last follow-up, } \\
\text { median (min; } \\
\text { max) (months) }\end{array}$ & $41.6(24 ; 63.5)$ & $44.8(29 ; 54.5)$ & .51 \\
\hline $\begin{array}{l}\text { Preoperative Tegner } \\
\text { score median } \\
\text { (min; max) }\end{array}$ & $7(4 ; 10)$ & $7.3(7 ; 9)$ & .56 \\
\hline Tibial slope $^{\circ} \pm$ SD & $11.6 \pm 3.6$ & $12.1 \pm 3.5$ & .42 \\
\hline $\mathrm{HKA}$ angle ${ }^{\circ} \pm \mathrm{SD}$ & $174.4 \pm 25.2$ & $176.4 \pm 4$ & .83 \\
\hline $\begin{array}{l}\text { Femoral tunnel } \\
\text { position } \% \pm \mathrm{SD} \\
\text { (Aglietti Ratio } \\
\text { method) }\end{array}$ & $77.2 \pm 3.6$ & $74.3 \pm 2.6$ & .89 \\
\hline $\begin{array}{l}\text { Tibial tunnel } \\
\text { position } \% \pm \mathrm{SD} \\
\text { (Aglietti Ratio } \\
\text { method) }\end{array}$ & $26.1 \pm 3.3$ & $23.7 \pm 1.6$ & .78 \\
\hline
\end{tabular}


Table 3. Comparison of Isokinetic Muscle Evaluation Results $\left(60^{\circ}\right.$ and $\left.180^{\circ} / \mathrm{s}\right)$ between the Two Groups (Student's $t$-Test)

\begin{tabular}{|c|c|c|c|}
\hline & $\underline{\text { Without ACLR Retears }}$ & $\underline{\text { With ACLR Retears }}$ & \\
\hline Isokinetic Test & $(n=93)$ & $(n=11)$ & $P$ \\
\hline $60^{\circ} / \mathrm{s}$ Healthy knee flexion $(\% / \mathrm{s} \pm \mathrm{SD})$ & $100.8 \pm 32.0$ & $90.8 \pm 27.4$ & .92 \\
\hline $60^{\circ} / \mathrm{s}$ Healthy knee extension $(\% / \mathrm{s} \pm \mathrm{SD})$ & $176.8 \pm 49.1$ & $159.0 \pm 40.2$ & .85 \\
\hline $60^{\circ} / \mathrm{s}$ Operated knee flexion $(\% / \mathrm{s} \pm \mathrm{SD})$ & $94.1 \pm 32.4$ & $66.1 \pm 26.2$ & .0415 \\
\hline $60^{\circ} / \mathrm{s}$ Operated knee extension $(\% / \mathrm{s} \pm \mathrm{SD})$ & $137.2 \pm 50.6$ & $87.8 \pm 46.6$ & .0225 \\
\hline $180^{\circ} / \mathrm{s}$ Healthy knee flexion $\left({ }^{\circ} / \mathrm{s} \pm \mathrm{SD}\right)$ & $79.4 \pm 27.1$ & $71.4 \pm 31.9$ & .91 \\
\hline $180^{\circ} / \mathrm{s}$ Healthy knee extension $\left({ }^{\circ} / \mathrm{s} \pm \mathrm{SD}\right)$ & $133.8 \pm 40.5$ & $120.1 \pm 41.5$ & .77 \\
\hline $180^{\circ} / \mathrm{s}$ Operated knee flexion $(\% / \mathrm{s} \pm \mathrm{SD})$ & $72.6 \pm 28.4$ & $61.2 \pm 24.2$ & .98 \\
\hline $180^{\circ} / \mathrm{s}$ Operated knee extension $(\% / \mathrm{s} \pm \mathrm{SD})$ & $106.9 \pm 40.2$ & $79.5 \pm 37.3$ & .79 \\
\hline $60^{\circ} / \mathrm{s}$ Operated/healthy flexion ratio $(\% \pm \mathrm{SD})$ & $95.0 \pm 17.5$ & $71.6 \pm 10.9$ & .0017 \\
\hline $60^{\circ} / \mathrm{s}$ Operated/healthy extension ratio (\% $\left.\pm \mathrm{SD}\right)$ & $81.8 \pm 32.0$ & $53.4 \pm 20.6$ & .035 \\
\hline $180^{\circ} / \mathrm{s}$ Operated/healthy flexion ratio $(\% \pm \mathrm{SD})$ & $92.1 \pm 20.7$ & $88.4 \pm 15.5$ & .7 \\
\hline $180^{\circ} / \mathrm{s}$ Operated/healthy extension ratio $(\% \pm \mathrm{SD})$ & $80.4 \pm 22.4$ & $65.5 \pm 19.6$ & .24 \\
\hline
\end{tabular}

NOTE. Bolded values indicate significant difference $(P<.05)$.

ACLR, anterior cruciate ligament reconstruction.

\section{References}

1. Holm I, Oiestad BE, Risberg MA, Aune AK. No difference in knee function or prevalence of osteoarthritis after reconstruction of the anterior cruciate ligament with 4strand hamstring autograft versus patellar tendon-bone autograft: A randomized study with 10-year follow-up. Am J Sports Med 2010;38:448-454.

2. Schmücker M, Haraszuk J, Hölmich P, Barfod KW. Graft failure, revision ACLR, and reoperation rates after ACLR with quadriceps tendon versus hamstring tendon autografts: A registry study with review of 475 patients. Am J Sports Med 2021;49:2136-2143.

3. Ahldén M, Samuelsson K, Sernert N, Forssblad M, Karlsson J, Kartus J. The Swedish National Anterior Cruciate Ligament Register: A report on baseline variables and outcomes of surgery for almost 18,000 patients. Am J Sports Med 2012;40:2230-2235.

4. Leroux T, Wasserstein D, Dwyer T, et al. The epidemiology of revision anterior cruciate ligament reconstruction in Ontario, Canada. Am J Sports Med 2014;42:2666-2672.

5. MOON Knee Group, Sullivan JP, Huston LJ, et al. Incidence and predictors of subsequent surgery after anterior cruciate ligament reconstruction: A 6-year follow-up study. Am J Sports Med 2020;48:2418-2428.

6. Hettrich CM, Dunn WR, Reinke EK, et al. The rate of subsequent surgery and predictors after anterior cruciate ligament reconstruction: Two- and 6-year follow-up results from a multicenter cohort. Am J Sports Med 2013;41: 1534-1540.

7. Chen H, Chen B, Tie K, Fu Z, Chen L. Single-bundle versus double-bundle autologous anterior cruciate ligament reconstruction: A meta-analysis of randomized controlled trials at 5-year minimum follow-up. J Orthop Surg Res 2018;13:50.

8. Samuelsen BT, Webster KE, Johnson NR, Hewett TE, Krych AJ. Hamstring autograft versus patellar tendon autograft for ACL reconstruction: Is there a difference in graft failure rate? A meta-analysis of 47,613 patients. Clin Orthop Relat Res 2017;475:2459-2468.
9. Bach BR. Revision anterior cruciate ligament surgery. Arthroscopy 2003;19:14-29.

10. Carson EW, Anisko EM, Restrepo C, Panariello RA, O'Brien SJ, Warren RF. Revision anterior cruciate ligament reconstruction: etiology of failures and clinical results. J Knee Surg 2004;17:127-132.

11. Legnani C, Zini S, Borgo E, Ventura A. Revision anterior cruciate ligament reconstruction with contralateral hamstring tendon grafts: 6 years follow-up. Joints 2017;5: 17-20.

12. Morgan JA, Dahm D, Levy B, Stuart MJ. Femoral tunnel malposition in ACL revision reconstruction. J Knee Surg 2012;25:361-368.

13. Trojani C, Sbihi A, Djian P, et al. Causes for failure of ACL reconstruction and influence of meniscectomies after revision. Knee Surg Sports Traumatol Arthrosc 2011;19: 196-201.

14. Alm L, Krause M, Frosch K-H, Akoto R. Preoperative medial knee instability is an underestimated risk factor for failure of revision ACL reconstruction. Knee Surg Sports Traumatol Arthrosc 2020;28:2458-2467.

15. Hodel S, Kabelitz M, Tondelli T, Vlachopoulos L, Sutter R, Fucentese SF. Introducing the lateral femoral condyle index as a risk factor for anterior cruciate ligament injury. Am J Sports Med 2019;47:2420-2426.

16. Lee JY, Kim HS, Heo ST, Kwon H, Jung S-N. Controlled continuous systemic heparinization increases success rate of artery-only anastomosis replantation in single distal digit amputation: A retrospective cohort study. Medicine (Baltimore) 2016;95:e3979.

17. Schlumberger M, Schuster P, Schulz M, et al. Traumatic graft rupture after primary and revision anterior cruciate ligament reconstruction: Retrospective analysis of incidence and risk factors in 2915 cases. Knee Surg Sports Traumatol Arthrosc 2017;25:1535-1541.

18. Tischer T, Paul J, Pape D, et al. The impact of osseous malalignment and realignment procedures in knee ligament surgery: A systematic review of the clinical evidence. Orthop J Sports Med 2017;5:23259671176 97287. 
19. Ardern CL, Webster KE. Knee flexor strength recovery following hamstring tendon harvest for anterior cruciate ligament reconstruction: a systematic review. Orthop Rev (Pavia) 2009; 1:e12.

20. Condouret J, Cohn J, Ferret J-M, et al. Évaluation isocinétique à deux ans de ligamentoplasties du ligament croisé antérieur au tendon rotulien et aux ischiojambiers. Rev Chir Orthop Répar Appar Moteur 2008;94(8, Supplement):375-382.

21. Nakamura N, Horibe S, Sasaki S, et al. Evaluation of active knee flexion and hamstring strength after anterior cruciate ligament reconstruction using hamstring tendons. Arthroscopy 2002;18:598-602.

22. Tashiro T, Kurosawa H, Kawakami A, Hikita A, Fukui N. Influence of medial hamstring tendon harvest on knee flexor strength after anterior cruciate ligament reconstruction. A detailed evaluation with comparison of single- and double-tendon harvest. Am J Sports Med 2003;31: 522-529.

23. Collette $\mathrm{M}$, Cassard $\mathrm{X}$. The tape locking screw technique (TLS): A new ACL reconstruction method using a short hamstring graft. Orthop Traumatol Surg Res 2011;97: 555-559.

24. Maffiuletti NA, Bizzini M, Desbrosses K, Babault N, Munzinger $\mathrm{U}$. Reliability of knee extension and flexion measurements using the Con-Trex isokinetic dynamometer. Clin Physiol Funct Imaging 2007;27:346-353.

25. Tegner Y, Lysholm J. Rating systems in the evaluation of knee ligament injuries. Clin Orthop Relat Res 1985;198: 43-49.

26. Aglietti P, Zaccherotti G, Menchetti PP, De Biase P. A comparison of clinical and radiological parameters with two arthroscopic techniques for anterior cruciate ligament reconstruction. Knee Surg Sports Traumatol Arthrosc 1995;3: 2-8.

27. Aglietti P, Buzzi R, Giron F, Simeone AJ, Zaccherotti G. Arthroscopic-assisted anterior cruciate ligament reconstruction with the central third patellar tendon. A 5-8year follow-up. Knee Surg Sports Traumatol Arthrosc 1997;5:138-144.

28. Undheim MB, Cosgrave C, King E, et al. Isokinetic muscle strength and readiness to return to sport following anterior cruciate ligament reconstruction: Is there an association? A systematic review and a protocol recommendation. Br J Sports Med 2015;49:1305-1310.

29. Barber-Westin SD, Noyes FR. Factors used to determine return to unrestricted sports activities after anterior cruciate ligament reconstruction. Arthroscopy 2011;27: 1697-1705.

30. Gokeler A, Welling W, Zaffagnini S, Seil R, Padua D. Development of a test battery to enhance safe return to sports after anterior cruciate ligament reconstruction. Knee Surg Sports Traumatol Arthrosc 2017;25:192-199.

31. Kyritsis P, Bahr R, Landreau P, Miladi R, Witvrouw E. Likelihood of ACL graft rupture: Not meeting six clinical discharge criteria before return to sport is associated with a four times greater risk of rupture. Br J Sports Med 2016;50:946-951.

32. Abrams GD, Harris JD, Gupta AK, et al. Functional performance testing after anterior cruciate ligament reconstruction: A systematic review. Orthop J Sports Med 2014;2: 2325967113518305.

33. Gobbi A, Mahajan S, Zanazzo M, Tuy B. Patellar tendon versus quadrupled bone-semitendinosus anterior cruciate ligament reconstruction: A prospective clinical investigation in athletes. Arthroscopy 2003;19:592-601.

34. Heijne A, Werner S. A 2-year follow-up of rehabilitation after ACL reconstruction using patellar tendon or hamstring tendon grafts: A prospective randomised outcome study. Knee Surg Sports Traumatol Arthrosc 2010;18:805-813.

35. Undheim MB, Cosgrave C, King E, et al. Isokinetic muscle strength and readiness to return to sport following anterior cruciate ligament reconstruction: Is there an association? A systematic review and a protocol recommendation. Br J Sports Med 2015;49:1305-1310.

36. Sengoku T, Nakase J, Asai K, et al. The effect of gracilis tendon harvesting in addition to semitendinosus tendon harvesting on knee extensor and flexor strength after anterior cruciate ligament reconstruction. Arch Orthop Trauma Surg. In press, doi:10.1007/s00402-021-03877-1.

37. Roger J, Bertani A, Vigouroux F, et al. ACL reconstruction using a quadruple semitendinosus graft with cortical fixations gives suitable isokinetic and clinical outcomes after 2 years. Knee Surg Sports Traumatol Arthrosc 2020;28: 2468-2477.

38. de Carvalho Froufe Andrade ACP, Caserotti P, de Carvalho CMP, de Azevedo Abade EA, da Eira Sampaio AJ. Reliability of concentric, eccentric and isometric knee extension and flexion when using the REV9000 isokinetic dynamometer. J Hum Kinet 2013;37: 47-53.

39. O'Connor RF, King E, Richter C, Webster KE, Falvey ÉC. No relationship between strength and power scores and anterior cruciate ligament return to sport after injury scale 9 months after anterior cruciate ligament reconstruction. Am J Sports Med 2020;48:78-84.

40. van Grinsven S, van Cingel REH, Holla CJM, van Loon CJM. Evidence-based rehabilitation following anterior cruciate ligament reconstruction. Knee Surg Sports Traumatol Arthrosc 2010;18:1128-1144.

41. Bodkin SG, Rutherford MH, Diduch DR, Brockmeier SF, Hart JM. How much time is needed between serial "return to play" assessments to achieve clinically important strength gains in patients recovering from anterior cruciate ligament reconstruction? Am J Sports Med 2020;48: 70-77.

42. Nagai T, Schilaty ND, Laskowski ER, Hewett TE. Hop tests can result in higher limb symmetry index values than isokinetic strength and leg press tests in patients following ACL reconstruction. Knee Surg Sports Traumatol Arthrosc 2020;28:816-822. 